\title{
Correlation between gamma analysis for midline and lateralized tumors by using volumetric modulated arc therapy
}

\author{
Syam Kumar Surendran Nair Ambika Devi', Anjana Parakkat Thokkayil², Aswathi Cheruparambil², \\ Sitha Gangadharan Padmini², Aparna Perumangat ${ }^{2}$ \\ ${ }^{I}$ Department of Radiation Oncology, Division of Radiation Physics, Malabar Cancer Centre Thalassery, Kerala, India \\ ${ }^{2}$ Department of Physics, University of Calicut, Kerala, India
}

Received April 23, 2015; Revised June 09, 2015; Accepted June 24, 2015; Published Online August 24, 2015

\section{Original Article}

\begin{abstract}
Purpose: The aim of this study was to evaluate the fluence for midline and lateralized tumors for volumetric modulated arc therapy (VMAT) by using a two-dimensional array. Methods: For this study, we selected 60 patients who were undergoing VMAT. The octavius phantom was computed tomography (CT) scanned and imported to the planning system. Verification plans were created for each plan and exported. The measurements were performed using 2D seven29 ion chamber array. Fluence measurement values for all the delivered plans were analyzed using VeriSoft software. The TPS calculated values were then compared with the measured gamma values. Results: The gamma pass percentage for midline tumors was found to be higher than that for lateralized tumors. The standard deviations between the gamma values for midline and lateralized tumors were 1.96 and 2.86, respectively. Moreover, the standard deviations between the point doses for midline and lateralized tumors were 0.360 and 0.283 , respectively. The mean gamma passing rate was $96.96 \%$ for midline tumors and $96.57 \%$ for lateralized tumors for 3\%DD/3-mm criteria. There is no significance found in the gamma values for midline and lateralized tumors with $p$-value 0.08. Conclusion: No particular correlation was found between the gamma pass percentage for midline tumors and that for lateralized tumors. Only a marginal difference was found in the gamma pass percentage.
\end{abstract}

Keywords: Quality Assurance; VMAT; 2D Seven29 Array; Octavius; Gamma Analysis; Midline Tumor

\section{Introduction}

The advantage of nonuniform conformal beams to target while sparing normal tissue has improved clinical outcome. Reducing the side effects to the nontarget organs permits tumor dose escalation and improves tumor control. The concept of volumetric modulated arc therapy (VMAT) was first proposed in 1995. VMAT is distinguished from intensity modulated radiation therapy (IMRT) in modulated fluence is achieved by a variable gantry speed, dose rate, and multileaf collimator (MLC) movements.

In this technique, the gantry moves continuously around the patient while the radiation is on. During rotation, the treatment field is also dynamically changed by the MLC movement. The success of intensity-modulated radiation delivery depends on the performance of the MLC, and its speed and position. Implementation of intensity-modulated beams has a complex dose distribution and a distinct advantage to the patient. Therefore, its application should be clinically validated. Historically, because of its complexity, IMRT plans have been dosimetrically verified in a phantom before treatment. Every patient plan is copied to the phantom geometry, and the dose is calculated from the treatment plan- ning system (TPS). The measured distribution is compared with the TPS-calculated distribution. TLD chips, diodes, films, and so on provide accurate measurement of dose distribution.

A patient-specific quality assurance (QA) procedure has also been implemented for VMAT. Quantitative analysis of patient-specific QA measurements is often used to determine whether the delivered field is appropriate for treating the patient. For point dose measurements, the percentage difference between the measured and planned doses is utilized. For planar dose measurements, a combination of the percent dose difference and distance to agreement (DTA) is typically used to reduce the analysis to a single metric.

For a stringent method of evaluating the dose calculated by using the TPS and the measured dose, a quantitative tool called gamma analysis $(\gamma)$ was developed by Low et al. ${ }^{1}$ This tool was initially developed by Harms et al. ${ }^{2}$ As an extension to the technique, Depuydt et al. ${ }^{3}$ and Low et al. ${ }^{4}$ proposed a metric called a gamma value that combines absorbed-dose difference and DTA criteria in a continuous distribution. The 
gamma value is a measure of how closely the calculated distribution matches the measured distribution, at a given measurement point. The gamma value will increase with DTA and the absorbed-dose difference between the measured and calculated values. The measurement is the reference information, and the calculated distribution is queried for comparison. ${ }^{5}$ It measures the closest distance between each reference point and the evaluated dose distribution after scaling by dose difference $(\Delta \mathrm{D})$ and distance to agreement $(\Delta \mathrm{d})$. The dose difference and DTA evaluations complement each other when used as determinants of agreement accuracy between the dose maps. ${ }^{6}$ This study aimed to evaluate the difference between the gamma values for midline tumors and those for lateralized tumors, and the effect of MLC movement on the pass percentage.

\section{Methods and Materials}

For this study, we selected 60 patients (which include 30 midline cases and 30 lateralized cases) who were undergoing VMAT. This includes medial and lateral tumors. Medial tumors refer to those situated at the midline of the body, and lateral tumors refer to those located toward the side or away from the midline of the body. Octavius computed tomography (CT) phantom was CT scanned and imported to the Eclipse version 10 TPS (Varian Medical Systems, Palo Alto, CA). Verification plans for midline and lateralized tumors were created separately by using the Varian Eclipse TPS with the 2D Seven29 detector array and the Octavius phantom (PTW, Freiburg, Germany).

\section{The octavius phantom}

The 2D Seven29 detector array system (PTW) comprises of an Octavius phantom (PTW), array interface, 2D Seven29 detector array, and software to obtain the data from the detector. The Octavius phantom used in this study is a dedicated phantom for QA of rotational treatments that focus primarily on the use of the Seven29 detector array. An octagonal shape was chosen to allow data acquisition in multiple planes with an easy phantom setup. It is made of polystyrene, with a physical density of $1.04 \mathrm{~g} / \mathrm{cm}^{3}$, a width of $32 \mathrm{~cm}$, and a length of $32 \mathrm{~cm}$. The bottom part of the phantom is removable and can accommodate a CT base or a linear accelerator (LINAC) base. The LINAC base has an air cavity to compensate for the reduced response when irradiated from the posterior side. A $30 \times 30 \times 2.2 \mathrm{~cm}^{3}$ central cavity allows inserting the ion chamber array into the phantom. The position of the cavity is such that when the 2D array is inserted, the plane through the middle of the ion chambers goes through the center of the phantom. ${ }^{4}$

\section{D ion chamber array}

The 2D-array Seven29 device is a two-dimensional detector array $(5.4 \mathrm{~kg})$ consisting of 729 cubic vented ionization chambers $\left(0.5 \times 0.5 \times 0.5 \mathrm{~cm}^{3}\right.$ each $)$ arranged in a $27 \times 27$ matrix with a center-to-center spacing of $10 \mathrm{~mm}^{2}$.The chambers are separated from each other by a 5-mm gap ${ }^{4}$. The upper electrode layer is positioned below a $0.5-\mathrm{cm}$ poly (methyl methacrylate) (PMMA) build-up layer .The lower electrode layer lies on top of a 2-mm thick electrode plate, which is again mounted on a $10-\mathrm{mm}$ PMMA base plate. The 5- and 10-mm PMMA layers have a water equivalent thickness of 0.59 and $1.18 \mathrm{~cm}$, respectively. The measurement ranges for the PTW 2D array is 200-1000 Gy and 8-500 $\mathrm{Gy} / \mathrm{min}$. The 2D array is calibrated for absolute dosimetry in a 60-Co photon beam at the PTW secondary standard dosimetry laboratory. ${ }^{5}$

\section{Pretreatment QA}

After the calculations, the verification plans for each patient was exported. Figure 1(a) shows the verification plan created for a patient with tumor at midline, and Figure1 (b) shows the verification plan created for lateralized tumors. Measurements were performed on a Varian Clinac 2100 iX, LINAC (Varian Medical Systems, Palo Alto, CA) equipped with a millennium 120-leaf collimator. The energy used was $6 \mathrm{MV}$, at a dose rate of $600 \mathrm{MU} / \mathrm{min}$. Verification plans for midline and lateralized tumors were separately delivered. The fluence measurements for all the delivered plans were analyzed by using the VeriSoft software (PTW). A gamma criterion of 3\% dose difference and 3-mm DTA has been used to evaluate the results. The TPS-calculated values were then compared with the measured gamma values. The point doses were also evaluated. ${ }^{7}$

\section{Gamma analysis}

Gamma analysis is a method for comparing two dose distributions. Dose difference and DTA are two parameters used in the assessment of dose distribution. A parameter that takes both dose deviation and DTA is the gamma index $(\gamma) .{ }^{8}$ Figure 2(a) shows the gamma analysis using VeriSoft for the TPS-calculated and array measurement values for midline tumors, and Figure 2(b) shows the analysis for lateralized tumors. The dose distribution comparison is subdivided into regions of high dose gradients and low dose gradients, each with different acceptance criteria. In the low-gradient regions, the doses are compared directly, with an acceptance tolerance placed on the difference between the measured and calculated doses. Since the dose difference in the high-dose gradient regions may be misleading, the concept of DTA is used. DTA is the reference data point and the nearest point in the compared dose distribution. ${ }^{3}$ 


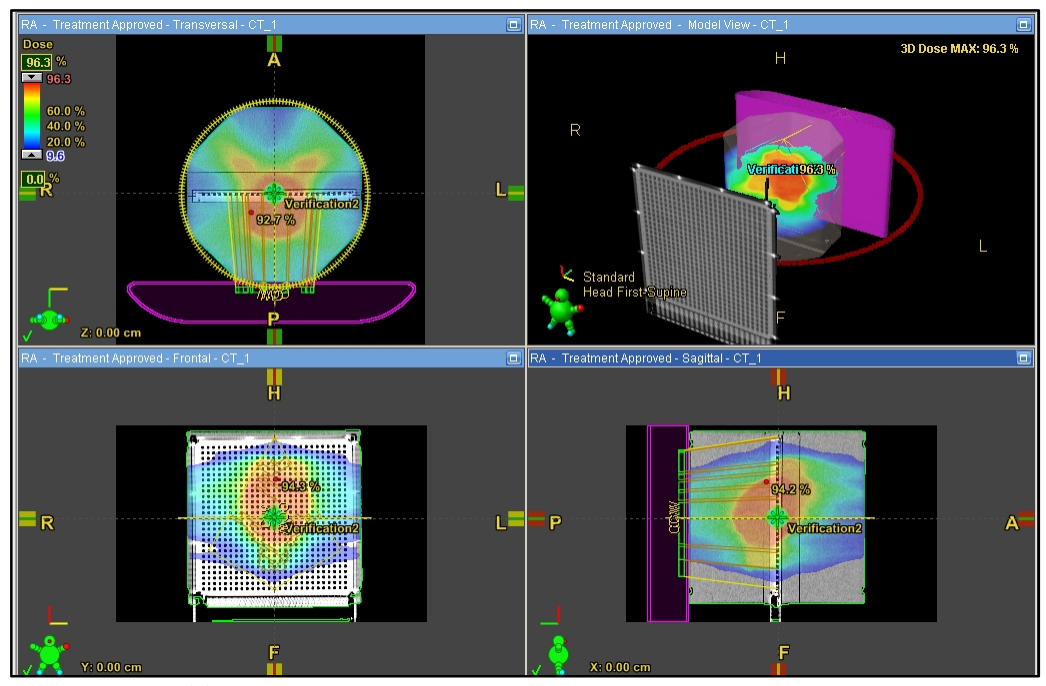

FIG. 1(a): Verification plans created for each patient for midline tumor.

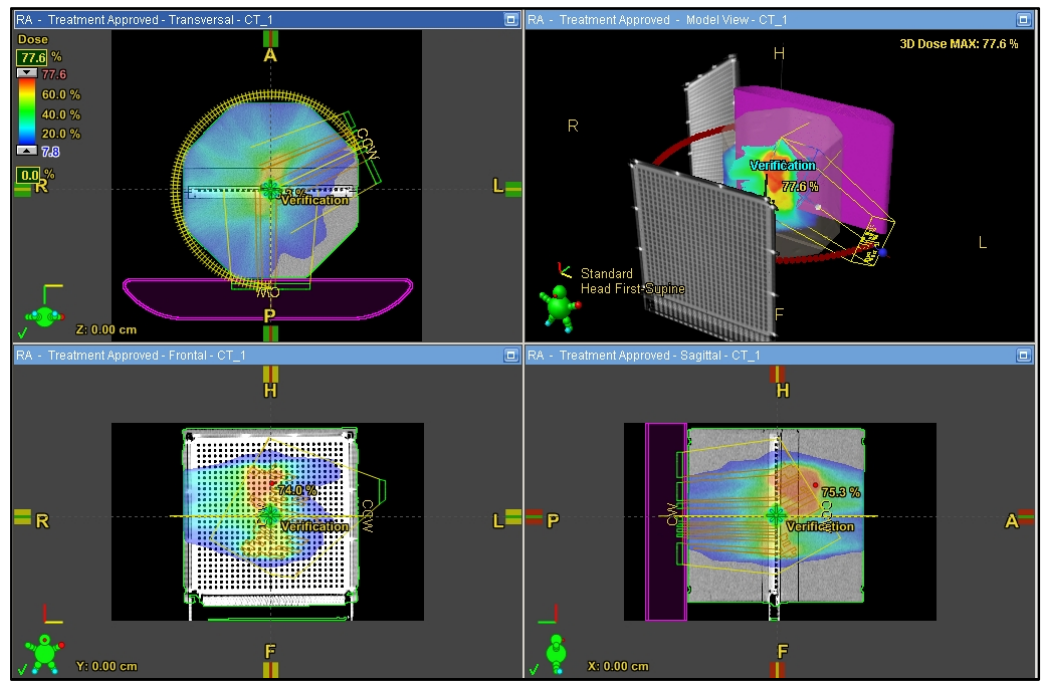

FIG. 1(b): Verification plans created for each patient for midline tumor for lateralized tumor.

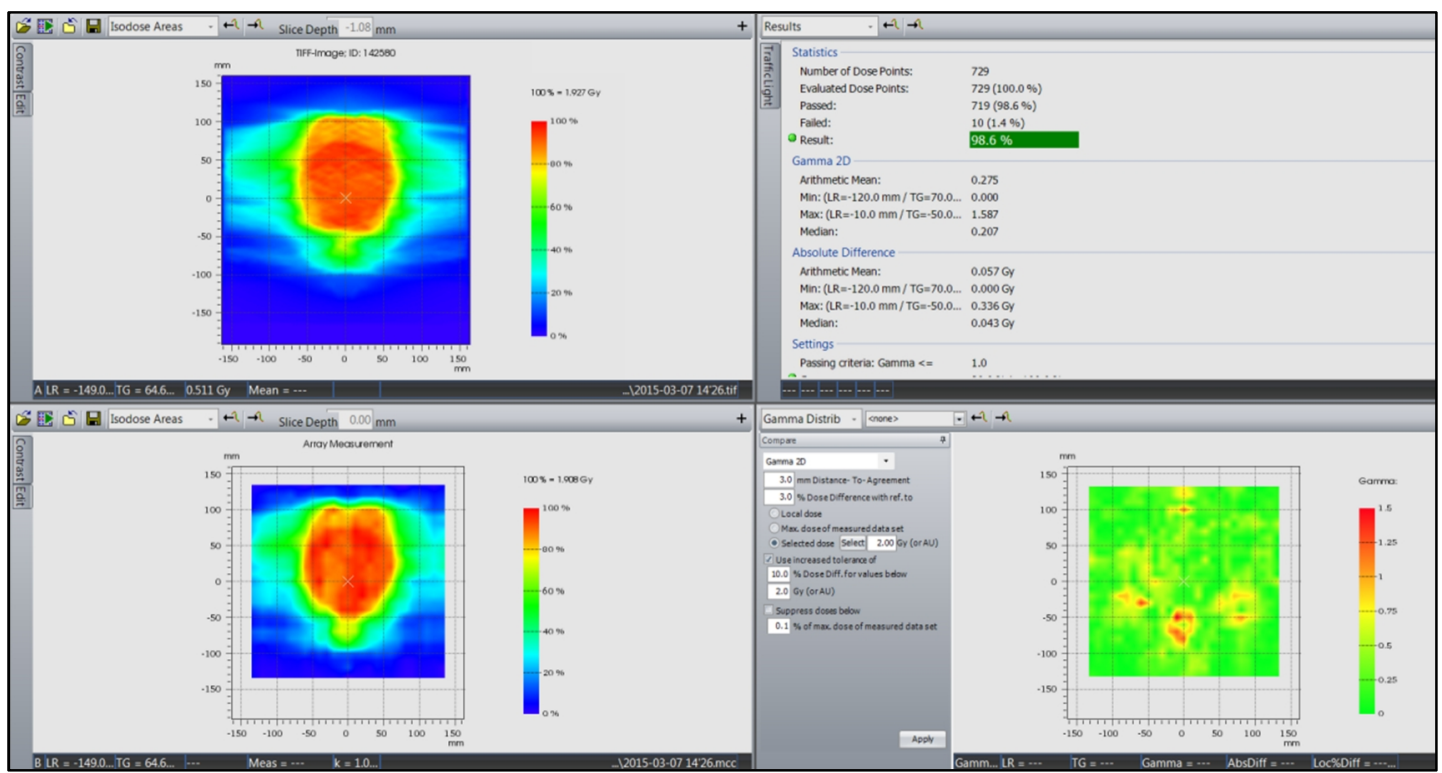

FIG. 2(a): The measured and calculated fluence for midline tumors were analysed using Verisoft Software, (PTW, Freiburg, Germany). 


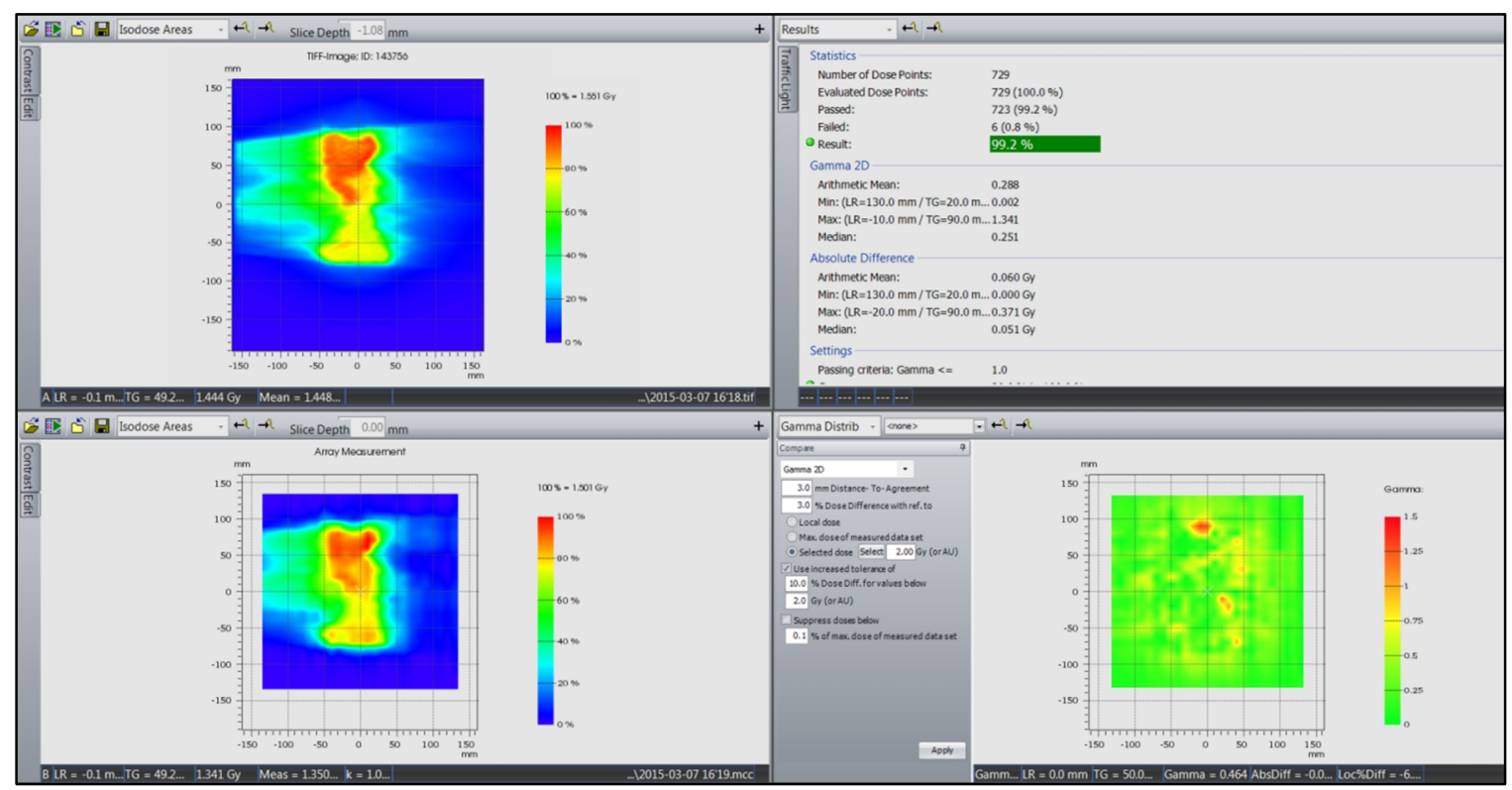

FIG. 2(b): The measured and calculated fluence for lateralized tumors were analyzed using Verisoft Software, (PTW, Freiburg, Germany).

\section{Results}

Previously, various studies have been conducted on 2D array by different authors. ${ }^{9-17}$ However; a clinical evaluation for the gamma analysis of midline and lateralized tumors has not been studied until now. The gamma pass percentage for midline tumors was found to be higher than that for lateralized tumors. The standard deviations between the gamma values for midline tumors and those for lateralized tumors were 1.96 and 2.86, respectively. Moreover, the standard deviations between the point doses for midline and lateralized tumors were 0.360 and 0.283 , respectively.

The average gamma passing rate was $96.96 \%$ for midline tumors and $96.57 \%$ for lateralized tumors for the $3 \% / 3-\mathrm{mm}$ criteria. The percentage variations for the midline and lateralized cases are shown in Figure 3(a) and (b). The Student $t$-test results showed no significant difference between the gamma pass percentage for midline tumors and that for lateralized tumors with $p=0.08$. For point doses, the Student $t$ test was performed to compare between the TPS-calculated and measurement values. For midline and lateralized tumors, the $p$-values were 0.48 and 0.47 , respectively, indicating no statistically significant difference. Figure 4 shows the percentage variation for (a) midline and (b) lateralized tumors. The discrepancy in the values obtained from the TPS calculation and array measurements may be due to the error during the entire process, such as errors in calculation, dosimetric measurement, and during delivery. The results showed that the pass percentage was slightly higher for midline tumors than for the lateralized ones. The gamma pass percentage, which is delivered by using the partial arc technique, was higher for lateralized tumors than for full-arc delivery. In addition, the standard deviation between the point doses was greater for midline tumors than for lateralized tumors. The average gamma pass percentage was almost the same for midline and lateralized tumors, according to the 3\% DD and 3-mm pass criteria.

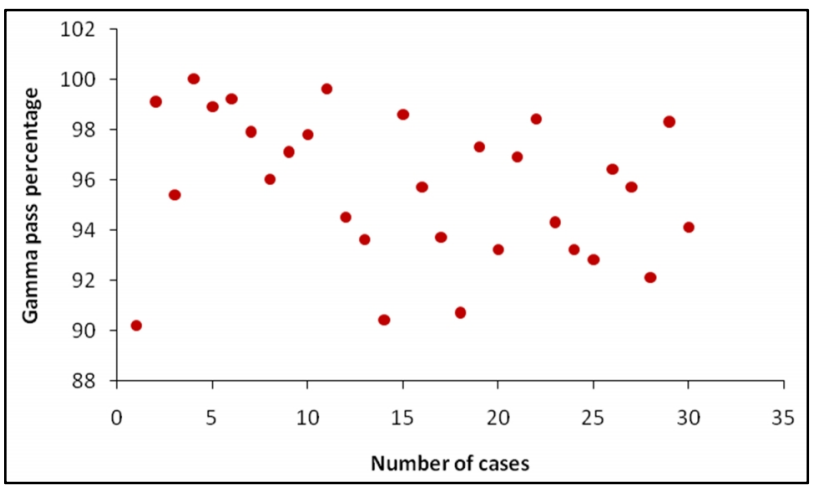

FIG. 3(a): Figure showing the gamma pass percentage for lateralized tumors.

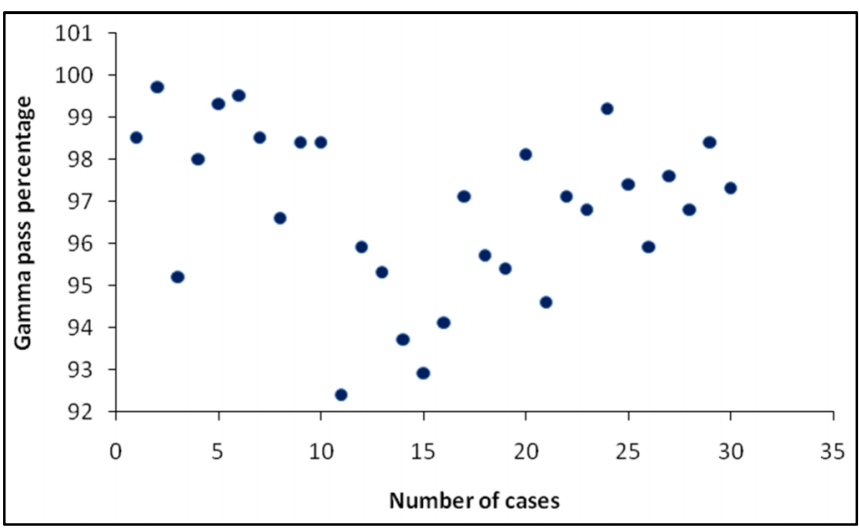

FIG. 3(b): Figure showing the gamma pass percentage for midline tumors. 


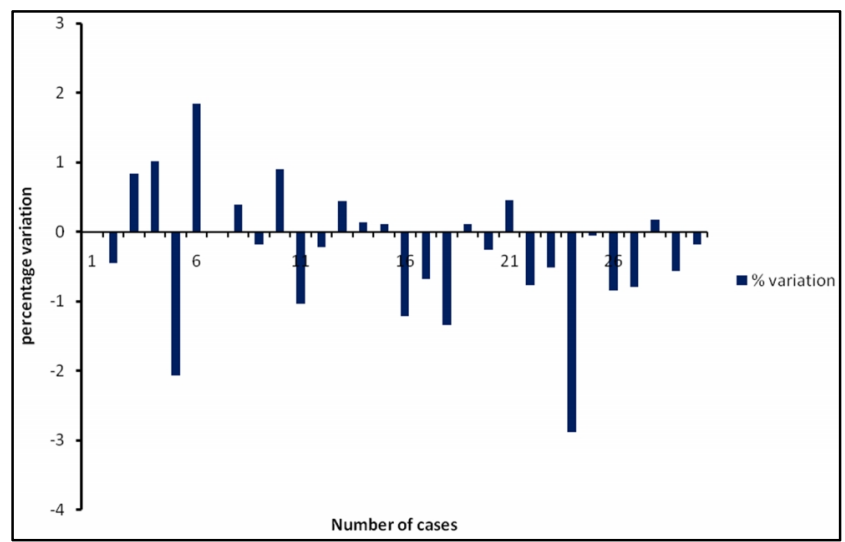

FIG. 4(a): Figure showing the percentage variation between TPS calculation and array measurement for midline tumors.

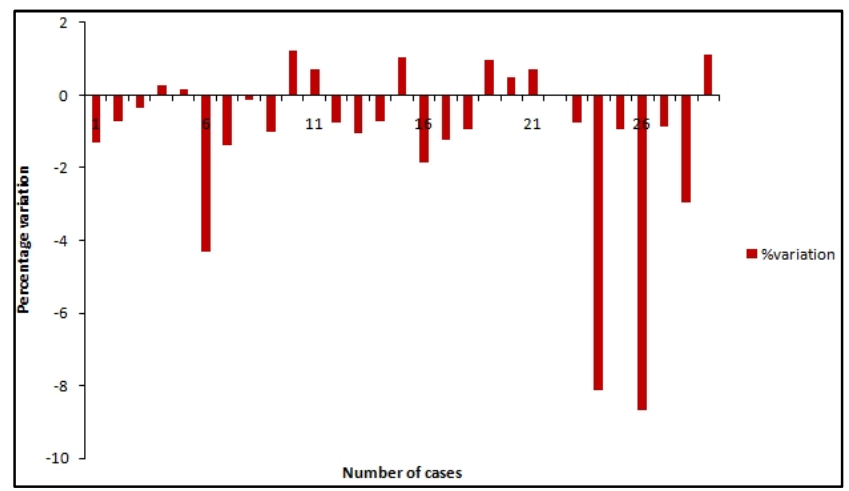

Figure 4(b): Figure showing the percentage variation between TPS calculation and array measurement for lateralized tumors.

\section{Discussion}

From the results obtained, only a slight difference in gamma pass percentage was found between midline and lateralized tumors. The standard deviation for midline tumors was less than that for lateralized tumors also the average gamma pass percentage for midline tumors was greater than that for lateralized tumors. Due to fewer critical structure constraints in midline tumor cases, the chance for high-pass percentage is higher. However, for lateralized tumors, a stringent constraint causes the MLC movement to become more complicated, which in turn reduces the pass percentage. For some lateralized tumor cases in which, the partial arc technique is used for treatment delivery the pass percentage is higher than the full-arc technique for lateralized tumors since many critical structures are avoided from the beam; thus, it increases the pass percentage. Even though the difference is negligible, the pass percentage for lateralized tumors could be increased by using IMRT technique or partial arc instead of full arc method.

\section{Conclusion}

From our analyses, we found no particular correlation between the gamma pass percentage for midline tumors and that for lateralized tumors. Only a marginal difference was observed in the gamma pass percentage for midline and lateralized tumors. Moreover, the values obtained had no significant difference in the $t$-test. More studies are to be conducted on this topic to make a more generalized conclusion. This study can be a pilot study in this topic.

\section{Conflict of interest}

The authors declare that they have no conflicts of interest. The authors alone are responsible for the content and writing of the paper.

\section{References}

1. Low DA, Dempsey JF. Evaluation of the gamma dose distribution comparison method. Med Phys. 2003;30:2455-64.

2. Harms WB Sr, Low DA, Wong JW, Purdy JA. A software tool for the quantitative evaluation of $3 \mathrm{D}$ dose calculation algorithms. Med Phys. 1998;25:1830-6.

3. Depuydt T, Van Esch A, Huyskens DP. A quantitative evaluation of IMRT dose distributions: refinement and clinical assessment of the gamma evaluation. Radiother Oncol. 2002;62:309-19.

4. Low DA, Harms WB, Mutic S, Purdy JA. A technique for the quantitative evaluation of dose distributions. Med Phys. 1998;25:656-61.

5. Kumar SA, Holla R, Sukumar P, et al. Treatment planning and dosimetric comparison study on two different volumetric modulated arc therapy delivery techniques. Rep PractOncolRadiother. 2012;18:87-94.

6. PTW Radiation Medicine QA Solutions, PTW, Freiburg, Germany.

7. Depuydt T, Van Esch A, Huyskens DP. A quantitative evaluation of IMRT dose distributions: refinement and clinical assessment of the gamma evaluation. RadiotherOncol. 2002;62:309-19.

8. Agazaryan N, Solberg TD, DeMarco JJ. Patient specific quality assurance for the delivery of intensity modulated radiotherapy. J ApplClin Med Phys. 2003;4:40-50.

9. Low DA. Gamma dose distribution evaluation tool. J Phys.: ConfSer2010;250: 012071.

10. Mancuso GM, Fontenot JD, Gibbons JP, Parker BC. Comparison of action levels for patient-specific quality assurance of intensity modulated radiation therapy and volumetric modulated arc therapy treatments. Med Phys. 2012; 39:4378-85.

11. Winiecki J, Morgaś T, Majewska K, Drzewiecka B. The gamma evaluation method as a routine $\mathrm{QA}$ procedure of IMRT.Reports of practical oncology and radiotherapy. 2009; 14:162-8. 
12. Prescribing, Recording, and Reporting Photon-Beam Intensity-Modulated Radiation Therapy (IMRT): Contents. J ICRU. 2010;10:NP.

13. Poppe B, Blechschmidt A, Djouguela A, et al. Twodimensional ionization chamber arrays for IMRT plan verification. Med Phys. 2006; 33:1005-15.

14. Li QL, Deng XW, Chen LX, et al. The angular dependence of a 2-dimensional diode array and the feasibility of its application in verifying the composite dose distribution of intensity-modulated radiation therapy. Chin J Cancer. 2010;29:617-20.
15. Chung H, Li J, Samant S. Feasibility of using two-dimensional array dosimeter for in vivo dose reconstruction via transit dosimetry. J ApplClin Med Phys. 2011;12:3370.

16. Mynampati DK, Yaparpalvi R, Hong L, et al. Application of AAPM TG 119 to volumetric arc therapy (VMAT). J ApplClin Med Phys. 2012;13:3382.

17. Van Esch A, Clermont C, Devillers M, et al. Online quality assurance of rotational radiotherapy treatment delivery by means of a 2D ion chamber array and the Octavius phantom. Med Phys. 2007; 34:3825-37. 